\title{
无氧气氛下糖溶液中 $\mathrm{Fe}(\mathrm{OH})_{2}$ 歧化 产物的穆斯堡尔谱学研究 ${ }^{*}$
}

\author{
张 经 坤 \\ (南开大学, 天津) \\ 刘荣川王述新 夏元复 \\ (南 京 大 学) \\ 衩守 仁 \\ (华中师范学院, 武昌)
}

在不同的溶液中, G. Schikorr ${ }^{[1]}$ 反应:

$$
3 \mathrm{Fe}(\mathrm{OH})_{2}=\mathrm{Fe}_{3} \mathrm{O}_{4}+2 \mathrm{H}_{2} \mathrm{O}+\mathrm{H}_{2},
$$

释放出的氢气量差别是很大的. Schrauzer ${ }^{[2]}$ 把溶液中不同的溶质称填加物, 且报道了不同填 加物对放氢影响的实验结果. 但并未阐述产生影响的原因. 我们在文献 [3,4]中已提出并论 证了关于这个反应产生氢的新的机理，本文是根据这个新的机理，通过对糖溶液中 $\mathrm{Fe}(\mathrm{OH})_{2}$ 歧化产物的穆斯堡尔谱学研究, 探讨糖类溶质对该反应放氢影响的原因.

\section{实验}

反应在 50-60m 的反应瓶中进行, 瓶口用血栓塞密封. 反应溶通过血栓塞注入. 瓶内充 有高纯氞气,并保持一个大气压. 所有的溶液均用二次蒸馏水新鮮配制, 并用流动氞气排除了 溶液中溶解的氧。 反应温度是 $40 \pm 0.5^{\circ} \mathrm{C}$ 、时间是 18 小时, $\mathrm{pH}$ 控制在 $9 \pm 0.1$. 沉淀过滤时均 在氯气保护下进行, 过滤后的沉淀压在黄铜槽里, 上面盖上两层聚乙烯薄膜, 封上石腊防止氧 化.

穆斯堡尔谱是在一台等加速谱仪上测定 ${ }^{[5]}$, 用的是 $10 \mathrm{mci} \mathrm{Co}^{5} / \mathrm{Pd}$ 的源．低温装置为金属 杜瓦瓶, 测试温度为 $80 \mathrm{~K}$. 在室温下, 用高纯 $\alpha-\mathrm{Fe}$ 标准样品标定速度. 实验所得的原始数 据,通过剥谱程序用 Lecroy-3500 型计算机系统剥去 $\mathrm{Fe}(\mathrm{OH})_{2}$ 谱线。

\section{结 果}

1. (a) D-果榶、(b) D-山梨糖和 (c) D-甘露糖的影响 这三种糖的浓度均配 成 $10 \%$, 各取其 $4.6 \mathrm{ml}$, 然后各加 $0.2 \mathrm{ml} 0.5 \mathrm{MFeSO}_{4}$, 再各加 $0.2 \mathrm{ml} 1 \mathrm{NNaOH}$, 在恒温槽内反 应. 反应完成后, 测其沉淀的穆斯堡尔谱. 如图 1 所示, $a 、 b 、 c$ 三个谱分别为从果糖、山梨 糖和甘露喏溶液中得到的沉淀所测的结果. 这三个体系放氢量均为零

本文 1982 年 8 月 15 日收到, 1983 年 7 月 21 日收到修改稿.

* 参加部分工作的还有南开大学倪音海, 南京大学朱琳, 江苏师院甘策强. 


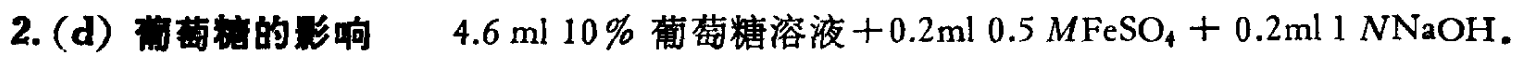
反应完成后对其沉淀所测得的穆斯堡尔谱示在图 1 中的 $\mathrm{d}$. 这个体系放氢为 $11.3 \mu \mathrm{Mol}$. 为了 叙述的方便，在下文中我们把四种糖简称为 $a 、 b 、 c 、 d$.

\section{讨 论}

1. 从图 1 中的四个谱看出, 在这四种糖溶液中 $\mathrm{Fe}(\mathrm{OH})_{2}$ 都发生了歧化,并给出了 $\mathrm{Fe}^{0[6]}$ 和 $\mathrm{Fe}_{3} \mathrm{O}_{4}{ }^{[7]}$ 的峰. 但 $\mathrm{a} 、 \mathrm{~b} 、 \mathrm{c}$ 这三个体系放氢量均为零. 这个结果再一次证明了我们在文献 [4]中 所提出的：氢的产生与歧化过程中产生的 $\mathrm{Fe}^{0}$ 没有直接关系; 发生歧化是产生氢的必要条件, 但不是充分条件. 这个新的观点是正确的. 我们在文献 [3] 中曾经报道了 $\mathrm{d}$ 的浓度对放氢作 用的影响，同样我们也对 $a 、 b 、 c$ 三种糖溶液做过 $5,10,15,20,25 \%$ 五种浓度条件下放氢的 实验, 其结果放氢量均为零. 它表明糖的浓度只是影响产生氢的多少问题,而糖本身的性质则 是决定氢能否产生的问题. a, b, c三体系均没产生氢，它说明这三种糖本身的性质抑制了产 生氢的充分条件,而 $\mathrm{d}$ 则满足了这个条件. 由此看来,糖的不同性质对放氢作用的影响差别是 很大的.

2. 为了更好地分析这四个谱,我们将每个谱中的 $\mathrm{Fe}(\mathrm{OH})_{2}$ 峰剥掉, 剥离后的谱表示在图 2 中, 并与图 1 中的 $a, b, c, d$ 一一对应. 每一谱线上的 $\mathrm{A}$ 谱线为超细颗粒的 $\mathrm{Fe}^{0}$ 的贡献 ${ }^{[6]}$, B 谱线为超细颗粒的 $\mathrm{Fe}_{3} \mathrm{O}_{4}$ 的贡献 ${ }^{[7]}$. 这些超细颗粒的 $\mathrm{Fe}^{0}$ 和 $\mathrm{Fe}_{3} \mathrm{O}_{4}$ 都呈单一谱线, 也就是 均应显出超顺磁性. 我们曾把这四个体系置于 3500 高斯磁场中进行反应, 测定了 $\Delta \mathrm{W}(\Delta \mathrm{W}$ 是整个体系在加磁场之后重量的变化量）与时间的关系. 当反应开始的瞬间（我们认为 $\mathrm{Fe}$

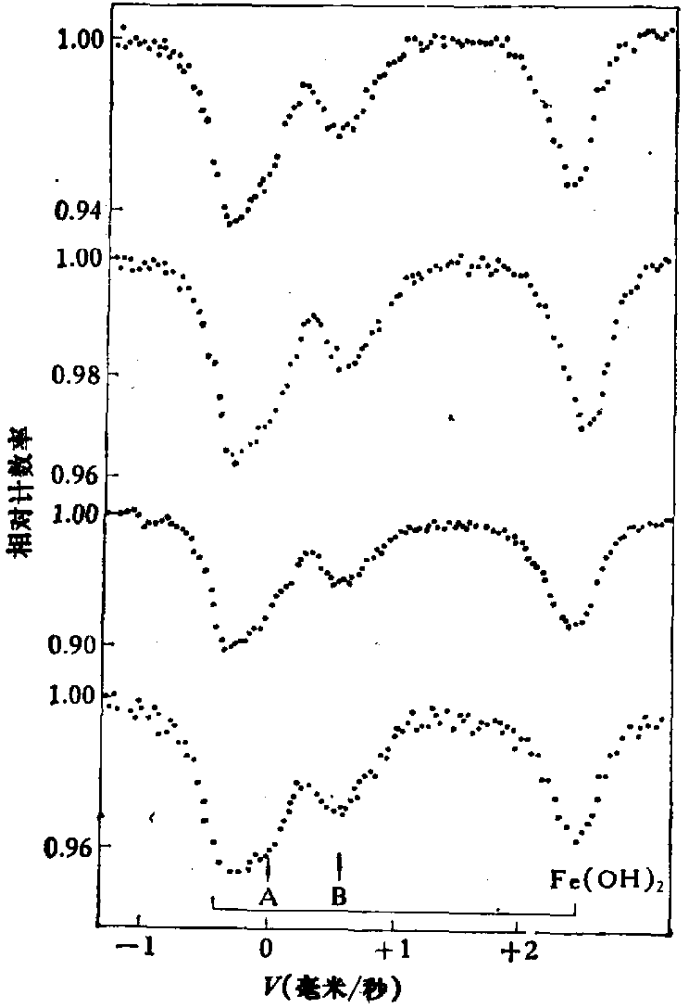

图 1 穆斯堡尔谱 $(80 \mathrm{~K})$

沉腚来自 (a)D-果糖, (b)D-山梨糖, (c)D-甘露糖, (d)D-甭萄糖溶液中

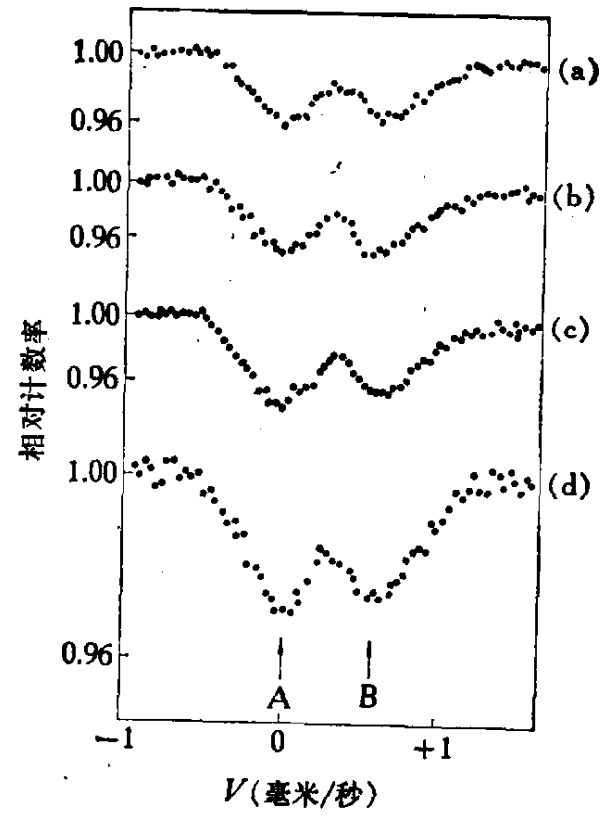

图 2 剥离去 $\mathrm{Fe}(\mathrm{OH})$ ）谱的穆斯堡尔谱并 与图 1一一对应 
$(\mathrm{OH})_{2}$ 尚没有发生歧化), 整个体系呈现出微小的抗磁性, 即 $\Delta \mathrm{W}<0$. 虽然按照结构理论或 晶体场理论 $\mathrm{Fe}(\mathrm{OH})_{2}$ 中的 $\mathrm{Fe}^{2+}$ 都应处高自旋态, 应表现出顺磁性, 但由于 $\mathrm{Fe}^{2+}$ 离子的重量 仅占总重量[样品管十反应溶]的万分之三,而玻玹和水都是抗磁性,所以总的磁性旺现出微弱 的抗磁性是合理的. 当反应进行 20 分钟后, 体系由抗磁性变成了顺磁性, 即 $\Delta \mathrm{W}>0$. 体系 磁性的改变, 只能是由于新生成的 $\mathrm{Fe}^{0}$ 和 $\mathrm{Fe}_{3} \mathrm{O}_{4}$ 具有超顼磁性所引起的. 这个结果与穆斯堡 尔谱所测定的结果是十分吻合的.

3. 从图 2 看出, 这四个图谱中的 $\mathrm{Fe}_{3} \mathrm{O}_{4}, \mathrm{Fe}^{0}$ 与 $\mathrm{Fe}(\mathrm{OH})_{2}$ 含量的相对比值差别是非常明 显的, 若令 $a$ 的 $\mathrm{Fe}_{3} \mathrm{O}_{4} / \mathrm{Fe}(\mathrm{OH})_{2}$ 的值为 1 , 则 $b, c$ 的值为 1.06 和 1.12, 而 $\mathrm{d}$ 的值却为 1.55. 这个结果完全可以用我们提出的放氢机理予以圆满的解释：在 $a, b, c$ 三体系内没有产生氢, 体系内的 $\mathrm{Fe}_{3} \mathrm{O}_{4}$ 完全是由于歧化而产生的, 而 $\mathrm{d}$ 体系内产生了氢, 即产生了 $2 \mathrm{Fe}^{2+}+2 \mathrm{H}^{+}=$ $2 \mathrm{Fe}^{3+}+\mathrm{H}_{2}$ 的反应, 所以它除了含有歧化产生的 $\mathrm{Fe}_{3} \mathrm{O}_{4}$ 外, 还有因发生放氢反应生成的 $\mathrm{Fe}^{3+}$ 而增加的一部份 $\mathrm{Fe}_{3} \mathrm{O}_{4}$, 它的相对含量比 $a, b, c$ 三体系内高是十分合理的. 而 $a, b, c$ 三体 系因都没有产生氢,反应条件又完全相同,所以相对含量十分相近.

4. 这四种糖的分子式完全相同, 即为 $\mathrm{C}_{6} \mathrm{H}_{12} \mathrm{O}_{6}$, 它们是同分异构体, 但它们对放氢作用的 影响差别这么大, 这只能从糖分子本身的结构和它们在溶液中所起的作用来考虑. 文献 [2] 认为填加物只起到分散的作用. 我们认为: 溶质的作用应由溶质的性质所决定. 就糖分子而 言,我们认为它将起到两个作用:（1）分散剂的作用: 当 $\mathrm{Fe}(\mathrm{OH})_{2}$ 刚发生沉淀时, 糖分子起 到一种分散剂的作用, 即把 $\mathrm{Fe}(\mathrm{OH})_{2}$ 分散成许许多多的小 “团粒”, 使 $\mathrm{Fe}(\mathrm{OH})_{2}$ 不能生成大 块结晶. 小 “团粒” 的大量形成增加了歧化中心，这要比没加任何溶质的从纯水中沉淀的 $\mathrm{Fe}(\mathrm{OH})_{2}$ 大大提高了歧化程度, 因而产生了许多的 $\mathrm{Fe}^{0}$ 和 $\mathrm{Fe}_{3} \mathrm{O}_{4}$. 虽然生成了许多的 $\mathrm{Fe}^{0}$ 和 $\mathrm{Fe}_{3} \mathrm{O}_{4}$, 但却因分散在被分散剂所隔开的小“团粒”中而不能形成大块结晶, 只能形成超细颗粒 的微晶,因而具有顺磁性,这是糖分子的第一个作用. (2) 络合剂的作用: 发生了歧化的小 “团 粒”，既有 $\mathrm{Fe}^{2+}$ 又含有 $\mathrm{Fe}^{3+}$, 对糖的分子具有很强的化学吸附作用, 被吸附的糖分子可以在 小“团粒”的局部发生络合作用，使小团粒形成多配位的“络合团粒”，我们简称它为“络团”，这 种“络团”在溶液中看得非常清楚. 反应完成后, 在瓶内出现了深绿色的絮状物, 悬浮在反应溶 液的中部, 除深绿色絮状物外, 其它部份呈现无色透明. 我们认为这种“络团”的稳定性, 就是 影响产生氢的直接原因.

$\mathrm{a}, \mathrm{b}$ 都是酮糖, $\mathrm{d}$ 是醛糖. 由于 $[\mathrm{R}-]$ 基的推电子作用, 在酮基 $[-\stackrel{O}{\mathrm{C}}-\mathrm{C}]$ 中的氧要比在 醛基 $[-\stackrel{O}{\mathrm{C}}-\mathrm{H}]$ 中的氧具有更大的负电性, 更容易提供 $2 \mathrm{p}$ 轨道上孤电子对去发生络合作 用. 同样条件下, 生成的络合物具有更大的稳定性. 所以 $a, b$ 溶液中的 “络团” 就十分稳定, 以致使 $\left[\mathrm{H}^{+}\right]$离子不能进入到“团粒”内部, 不能发生放氢反应. 而 $\mathrm{d}$ 溶液中的“络团”就不那么 稳定, $\left[\mathrm{H}^{+}\right]$离子就能够进入“团粒”内部发生放氢反应, 放出氢气. 至于甘露糖也是醛糖, 在 这种条件下为什么不放氢? 我们认为这可能是由于甘露糖与葡萄糖的空间构型不同，因而生 成的“络团”的稳定性大于葡萄糖生成的“络团”稳定性 (关于这个问题,另文讨论)，但这种作用 应低于 $[-\stackrel{\mathrm{O}}{\mathrm{C}}-]$ 的作用,为了证实这种观点,我们做了两项实验:（1）改变 $\mathrm{pH}$ 值, 观察“络 
团”的稳定性, 保持其它条件不变, 把 $\mathrm{NaOH}$ 的用量增加到 $0.22,0.24,0.26,0.28,0.32 \mathrm{ml}$, 随着 $\mathrm{pH}$ 值的增加, $\mathrm{a}, \mathrm{b}, \mathrm{c}$ 三体系絮状物不断减少, 当 $\mathrm{pH}$ 增大到 10.5 以后, 絮状物几乎消 失,反应溶液变成了深绿色的真溶液; 而 $\mathrm{d}$ 体系随着 $\mathrm{pH}$ 值的增加, 絮状物也不断消失, 但反应 溶液底部却出现大量沉腚. 这说明 $\mathrm{a}, \mathrm{b}, \mathrm{c}$ 三体系随着 $\mathrm{pH}$ 值的增加“络团”更加稳定, 而 $\mathrm{d}$ 则 相反.（2)降低 $a, b, c$ 三种“络团”的稳定性,使它们也产氢: 我们认为既然 $a, b, c$ 三体系内 $\mathrm{Fe}(\mathrm{OH})_{2}$ 也发生了相当程度的歧化,它们也就具备了产生氢的必要条件,如果设法降低这三种 “络团”的稳定性,增大它们的解离度,使 $\left[\mathrm{H}^{+}\right]$有可能进人 “团粒” 内部，那么它们也应该产生 出氢. 我们采取了两种办法, (A)加人 $\left[\mathrm{Ni}^{2+}\right]$ 离子, 使 $\mathrm{Fe}(\mathrm{OH})_{2}$ 与 $\mathrm{Ni}(\mathrm{OH})_{3}$ 共晶, 减慢歧化速 度 ${ }^{[4]}$ ，(B) 降低 $\mathrm{pH}$ 值,这样一则降低了“络团”的稳定性, 二则增加了 $\left[\mathrm{H}^{+}\right]$离子的浓度. 实验 结果指出：当 $\mathrm{pH}$ 值控制在 $5.8 \pm 0.1$ 时,这三种体系均产生了氢,分别为 (a) $1.258 \mu \mathrm{Mol}$ ，(b) $6.145 \mu \mathrm{Mol}$ ，(c) $11.875 \mu \mathrm{Mol}$ 。这个实验结果首先证明了“络团”的存在,即证明了我们提出的 关于糖分子的第二个作用的观点是正确的. 其次，(c) 体系放的氢远高于 (a) 和 (b) 体系, 也证明了我们认为空间构型的影响低于 $[-\stackrel{\text { C }}{\mathrm{C}}-]$ 的影响的假设是合理的.

关于不同溶质对放氢作用的影响,我们已在文献 [3] 中报道了实验结果,并将另文继续予 以讨论. 但总的来讲, 我们认为: 溶质一定程度上都具有分散剂的作用, 至于影响放氢的作 用, 因溶质的性质的不同而不同. 就糖类而言, 我们认为溶液中的糖分子与 $\mathrm{Fe}^{2+}\left(\mathrm{Fe}^{3+}\right)$ 生成络 合物的稳定性,是影响产生氢的直接原因.

\section{考文献}

[1] Sehikorr, G., Z. Elektrochim., 35 (1929), 65.

[ 2 ] Schrauzer, G. N. \& Guth, T. D., J. A. C. S., 99(1977), 7189.

[3] 张经坤等,高等学校化学学报, 1982, 4: 568 .

[4] 张经坤等,科学通报, 28(1983), 2: 91 .

[5] 南京大学穆斯堡尔谱科研组,南宗大学学报, 1978, 1: 48 .

[6] Boudart, M. et al., J. Acta, $37(1970), 486$.

[ 7 ] MeNab, T. K. et al., J. Appl. Phys., 39(1968), 5703. 